\title{
BILATERAL THALAMIC GLIOMA -A RARE TUMOUR
}

\author{
Vivek Rauniyar, Bing Qin, Huan Yang
}

\begin{abstract}
We report a case of 48-year old woman who presented with dementia, progressive mental decline, personality change, paresis of the right lower extremity, and gait ataxia. Magnetic resonance imaging (MRI) T1-weighted image revealed bilateral thalamic swelling with homogenous low signal intensity and mild contrast enhancement with gadolinium. The T2weighted image showed high intensity lesions in the bilateral thalamus, septum pellucidum and fornix. A CT-guided stereotactic biopsy of the lesion revealed fibrillary astrocytoma grade III as per WHO classification. Bilateral thalamic gliomas are very rare tumours and when present may resemble other benign diseases having similar clinical presentations.
\end{abstract}

Key words: Thalamic glioma; stereotactic biopsy; fibrillary astrocytoma;

\section{Introduction}

Gliomas of astrocytic, oligodendroglial and ependymal origin account for more than $70 \%$ of all the brain tumors (1). However, primary thalamic tumors are rare and bilateral thalamic gliomas are even more rare (2). Thalamic astrocytomas are not easily diagnosed as their clinical presentation are often nonspecific and conventional neuroimaging findings are not typical for the tumors, and can be easily mistaken for other diseases $(3,4,5)$. Dementia, personality changes, cognitive impairments with or without paresis are presenting clinical features of the bilateral thalamic gliomas in adults (6) and can mimic other infective, demyelinating, and inflammatory disorders. It might require a high degree of suspicion and invasive diagnostic test to arrive at correct diagnosis.

\section{Case report}

A 48-year-old woman was admitted to our department with complaints of progressive memory loss, personality changes, dizziness, ataxia and weakness in her extremities for five months. Two months back, she was treated at local county hospital suspected of having neurosyphillis as she was serum positive for anti-treponemal antibody. She did not improve with the treatment and landed at our hospital.

On admission to our department, her examination revealed memory impairment, decreased spontaneous eye movement, paresis of the right extremity, and gait ataxia. On brief neuropsychiatric assessment, she had MMSE score of 14/30 along with obvious mild to medium cognitive impairment, recent memory and recall loss. Language expression, comprehension and calculation abilities were also found to be mildly affected. The routine laboratory tests of blood, serum biochemistry, urine, and stool failed to reveal any abnormality and serum anti-treponemal antibody was negative. The cerebrospinal fluid was clear, colourless, with a mild increase of $\operatorname{IgG} \quad 0.05 \mathrm{mg} / \mathrm{L}$ (normal $0-0.03$ 
$\mathrm{mg} / \mathrm{L}$ ), along with a normal opening pressure, cell count, protein, and glucose and negative for cytology, anti-treponemal antibody. EEG showed diffuse nonspecific slow waves. MRI T2 weighted image showed high intensity at bilateral thalamus, septum pellucidum and fornix. (Fig.1).

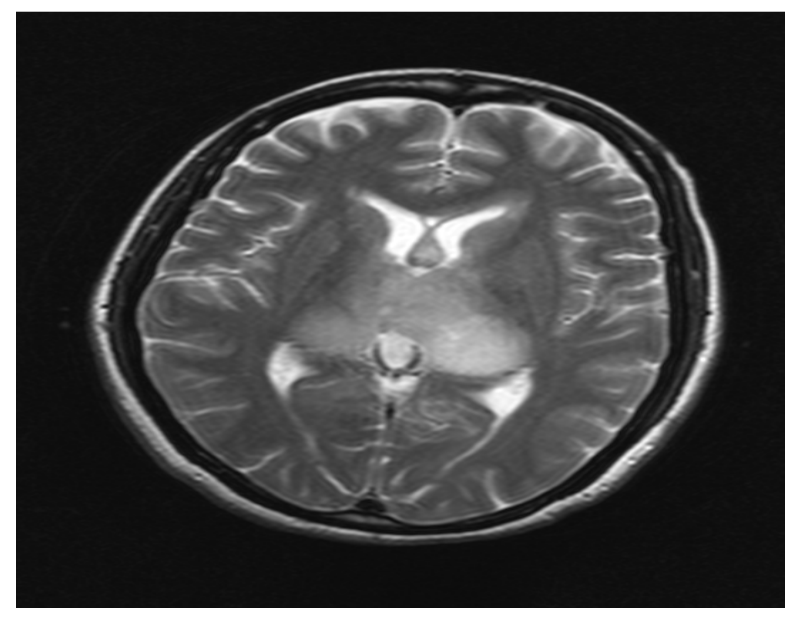

Figure 1

T2 coronal image at the level of thalamus showing bilateral thalamic glioma

Slight enhancement of these lesions could be observed with Gd-DTPA (Fig.2) and MRA was normal.

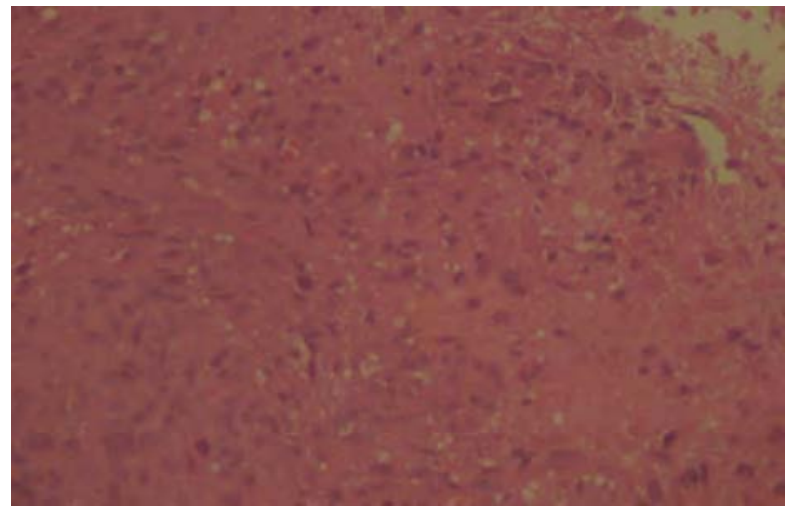

Figure 2

Biopsied tissue of Bilateral Thalamic Glioma (grade III) on histopathological examination.

A CT-guided stereotactic biopsy was performed with consultation of neurosurgeons which revealed prominent proliferation of fibrillary astrocytes, mitoses, pleomorphic nuclei, and vascular endothelial hyperplasia on histopathological examination consistent with the fibrillary astrocytoma grade III as per WHO classification (fig3).

She was transferred to neurosurgery department for therapeutic work-up.

\section{Discussion}

Bilateral thalamic involvement is rare, nonetheless, can be found in various types of benign as well as malignant disorders such as viral infections, bacterial infections, demyelinating disorders, metabolic diseases, and degenerative diseases (7). There is a long list of differential diagnoses of benign disorders that can affect the thalamus bilaterally.And intriguingly these benign disorders have similar clinical presentations. Interestingly, because the thalamus is affected bilaterally, the symptom complex is not typical for any disorder. Neurosyphillis may present with bi-thalamic lesions and the most common manifestations are the personality changes and memory impairment (8).Similarly, Viral infections caused by the flavivirus family-e.g. Japanese encephalitis virus and others typically affects the subcortical gray matter including the thalamus bilaterally (9).Likewise, tuberculous meningo-encephalitis, malaria have been rarely reported presenting with bilateral thalamic lesions (10,11).Also Acute Demyelinating Encephalomyelitis (ADEM) has been reported with bilateral thalamic lesions in $40 \%$ of ADEM cases (12).

The principle clinical manifestations in our case were dementia, personality change, 
cognitive deficits, paresis of the right extremity, and gait ataxia .Personality changes and mental deterioration possibly indicate the destruction of medial and intralaminar nuclei of thalamus(13). Similarly paresis of the right extremity and gait ataxia are common finding in thalamic tumours and may indicate the involvement of the dorsomedial nuclei of the thalamus which project to prefrontal area(13). Though thalamic tumours are usually associated with increased intracranial pressure(14), in our subject there were no signs of raised ICP.

Hence, the case we have presented here exhibits a rare clinical encounter and illustrate the rarity and difficulty of diagnosing rare bilateral thalamic gliomas.

\section{References}

1. Ohgaki H. , Kleihues P., Epidemiology and etiology of gliomas. Acta Neuropathol. 2005;109:93-108.

2., Carpio-O’Donovan R, Melanson D, Peters TM (1992) Bilateral thalamic glioma: review of eight cases with personality change and mental deterioration. Am J Neuroradiol. 13:1225-1230.

3. Krouwer HGJ, Prados MD (1995) Infiltrative astrocytomas of the thalamus. J Neurosurg. 82:548-557.

4. Rogers LR, Weinstein MA, Estes ML, Cairncross JG, Strachan T (1994) Diffuse bilateral cerebral astrocytomas with atypical neuroimaging studies. J Neurosurg. 81:817-821.

5. Russell DS, Rubinstein LJ (1989) Pathology of tumors of the nervous system, 5th edn.Arnold, London, Melbourne Auckland
6. S. Nishio, T. Morioka, S. Suzuki, I. Takeshita, M. Fukui. Thalamic Glioma: a clinicopathologic analysis of 20 cases with reference to patient age. Acta Neurochirurgica(wien) (1997) 139:336-342.

7. Jennifer L., Adrian D., Lisa Ann H.,Klaus C. S., Hartmut B., Differential diagnosis of Bilateral Thalamic Lesions. Clin. Neuroradiol. 2007; 17:3-22.

8. Roberts M. C., Emsley R.A.,Psychiatric manifestations of Neurosyphillis. SAMJ 1992 Nov. (82):335-37.

9. Kalita J, Misra UK. Comparison of CT scan and MRI findings in the diagnosis of Japanese encephalitis. J Neurol Sci. 2000; 174:3-8.

10. Wakai M, Hayashi M, Honda $K$, et al. Acute onset of tuberculous meningoencephalitis presenting with symmetric linear lesions in the bilateral thalamus: a case report. Rinsho Shinkeigaku 2001;41:519-22.

11. Cordoliani YS, Sarrazin JL, Felten D, et al. MR of cerebral malaria. AJNR Am J Neuroradiol 1998;19:871-4.

12. Garg RK. Acute disseminated encephalomyelitis. Postgrad Med J 2003; 79:11-7.

13. Uchino M, Kitajima S, Miyazaki C, Shibata I, Miura M. Bilateral thalamic glioma-case report. Neurol Med Chir (Tokyo). 2002 Oct;42(10):443-6.

14.Cheek WR., Taveras M.Thalamic tumors.J Neurosurgery 24:505-513,1966.

15. Franc,ois Este`ve, Sylvie Grand, Christophe Rubin, Dominique Hoffmann, Basile Pasquier, Danielle Graveron-Demilly, Rachid Mahdjoub, and Jean-Franc, ois Le Bas. MR Spectroscopy of Bilateral Thalamic Gliomas. AJNR Am J Neuroradiol May 1999;20:876-881.

Corresponding Address: Dr Huan Yang, MD. Department of Neurology, Xiangya Hospital, Central South University, Changsha, Hunan, 410008, China Email: yangh69@yahoo.com, Tel.: +86731 4327236; fax: +867314327401. 